QUELLE EST LA VALEUR DU GENRE THYSANOT AENIA BEDDARD, 1911 ? (A PROPOS D'UNE REDESCRIPTION DE T. LEMURIS BEDDARD, ANOPLOCEPHALIDAE DE MADAGASCAR)

\author{
Par S. DEBLOCK et A. DIAOURÉ
}

Hôte :

Lemur macaco $L$. Intestin grêle.

Localisation géographique :

Madagascar.

Date de récolte:

1958 (Pr A.-G. Chabaud leg.).

Matériel d'étude:

Six Cestodes peu matures; un Cestode comportant des anneaux mûrs, et un fragment de chaîne gravide (21 anneaux); coupes longitudinales et transverses.

\title{
Description de l'espèce.
}

Taille : longueur totale : $72 \mathrm{~mm}$. environ (avec les derniers anneaux mûrs : 8 à $9 \mathrm{~cm}$. vraisemblablement).

La largeur s'accroît régulièrement depuis le cou jusqu'aux anneaux les plus mûrs ; largeur maximale : $2,6 \mathrm{~mm}$. à $3 \mathrm{~mm}$.

Scolex (cf. fig. 1).

Bien développé. Dépourvu de rostre. Inerme. De forme générale massuée à extrémité antérieure un peu aplatie. Largeur maximale au niveau des ven. touses : 580 à $760 \mu$ (moyenne : $680 \mu$ ). Sa partie postérieure s'amincit très progressivement pour se continuer sans limitation nette avec le cou. Pourvu de quatre ventouses rondes ou ovalaires disposées deux à deux sur chaque face, à ouvertures latérales ou légèrement antéro-latérales; saillantes à la surface du scolex. Diamètre : $260-360 \times 260-290 \mu$. Epaisseur de leur bord musculaire : $60 \mu$ environ.

\section{Cou.}

Long. Allant en s'amincissant très progressivement à partir du scolex pour s'élargir à nouveau dès qu'apparaissent les premières marques de segmentation 
à environ $1.500 \mu$ du bord inférieur des ventouses ; sa largeur minimale est de 330-380 $\mu$ (moyenne : $360 \mu$ ).

\section{Strobile.}

Il doit compter de 150 à 200 anneaux au total. Les anneaux jeunes sont beaucoup plus larges que longs (exemple : $1.725 \mu \times 450 \mu$ à $25 \mathrm{~mm}$. du scolex) et jamais imbriqués les uns dans les autres, à la différence des anneaux mûrs qui le sont légèrement. Puis leur longueur s'accroît progressivement, sans

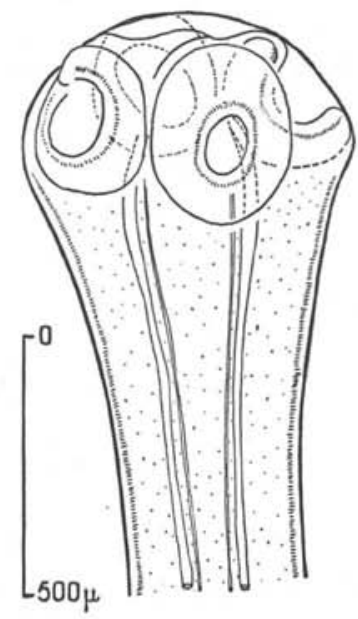

Fig. 1, - Scolex

dépasser la valeur de la largeur. Les bords latéraux sont très convexes, le bord postérieur est rectiligne, dépourvu de franges.

\section{Cuticule.}

Epaisse de $6,5 \mu$, dépourvue d'ornementation.

\section{Système excréteur.}

Constitué classiquement de deux paires de canaux longitudinaux. Les vaisseaux ventraux, d'un calibre important (de $34 \mu$ dans les anneaux immatures à $80 \mu$ dans les anneaux mûrs, où s'individualise leur paroi plus colorable), sont, le plus souvent, externes par rapport aux canaux dorsaux d'un calibre beaucoup plus réduit $(9-10 \mu)$. Ils courent à environ $290-340 \mu$ du bord de l'anneau mûr.

A la partie postérieure de chaque anneau, deux anastomoses transverses, l'une dorsale, l'autre ventrale, réunissent chacune des paires longitudinales homologues. De plus, il peut exister dans certains anneaux un réseau peu dense de canalicules secondaires dorsaux compliquant les relations entre les canaux longitudinaux et transverses. L'anastomose transverse dorsale et le réseau disparaissent dans les anneaux mûrs, ou ne sont plus visibles. 
La position fondamentale des canaux osmo-régulateurs paraît être de part et d'autre des conduits sexuels; mais, dans $10 \%$ des cas environ, le canal ventral les franchit dorsalement. En fait, la fréquence de l'anomalie est variable avec les strobiles ainsi qu'il ressort du tableau suivant :

\begin{tabular}{|c|c|c|c|c|c|}
\hline \multirow{3}{*}{$\begin{array}{c}\text { Strobiles } \\
\qquad \mathrm{N}^{\circ}\end{array}$} & \multirow{3}{*}{$\begin{array}{c}\text { Nombre } \\
\text { d'anneaux } \\
\text { successifs } \\
\text { observés }\end{array}$} & \multicolumn{4}{|c|}{ canal excréteur } \\
\hline & & \multicolumn{2}{|c|}{ ventral } & \multicolumn{2}{|c|}{ dorsal } \\
\hline & & $\begin{array}{c}\text { en } \\
\text { position } \\
\text { ventrale }\end{array}$ & $\begin{array}{c}\text { en } \\
\text { position } \\
\text { dorsale }\end{array}$ & $\begin{array}{c}\text { en } \\
\text { position } \\
\text { ventrale }\end{array}$ & $\begin{array}{c}\text { en } \\
\text { position } \\
\text { dorsale }\end{array}$ \\
\hline 1 & 34 & 33 & 1 & 34 & 0 \\
\hline 2 & 17 & 14 & 3 & 17 & 0 \\
\hline 3 & 13 & 13 & 0 & 13 & 0 \\
\hline 4 & 13 & 12 & 1 & 13 & 0 \\
\hline 5 & 17 & 17 & 0 & 17 & 0 \\
\hline 6 & 14 & 9 & 5 & $\begin{array}{l}\text { Invi- } \\
\text { sibles }\end{array}$ & 0 \\
\hline
\end{tabular}

\section{Nerf.}

Bien visible. Sa position par rapport aux conduits sexuels est indifférente (sur la série de 34 anneaux citée précédemment, on le trouve 15 fois dorsal et 19 fois ventral).

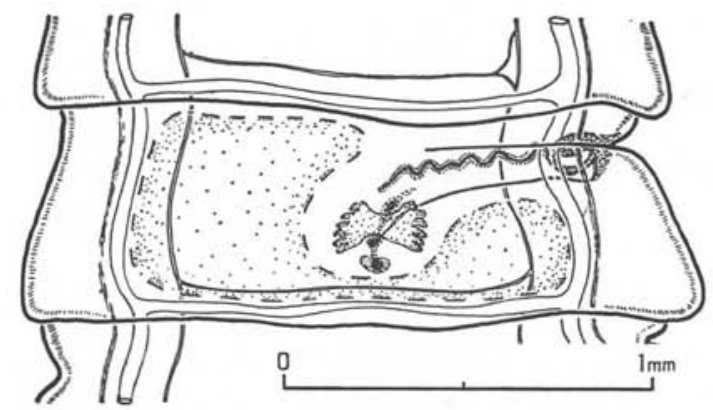

Fig. 2. - Anneau immature, vue ventrale $\left(100^{\circ}\right.$ anneau de la chaîne). Le champ testiculaire est délimité par le trait discontinu bordant le pointillé. 


\section{Système musculaire.}

Le système musculaire longitudinal est fortement développé, constitué de nombreuses fibres isolées qui, de rares et fines à la périphérie corticale, deviennent de plus en plus fortes et nombreuses au fur et à mesure qu'elles se rapprochent de la limite du parenchyme médullaire où elles forment une nappe continue.

Anneaux immatures (cf. fig. 2).

Nombre : une centaine (y compris les anneaux du cou) sur une longueur de $40 \mathrm{~mm}$. environ. Les massifs cellulaires destinés à fournir les glandes génitales

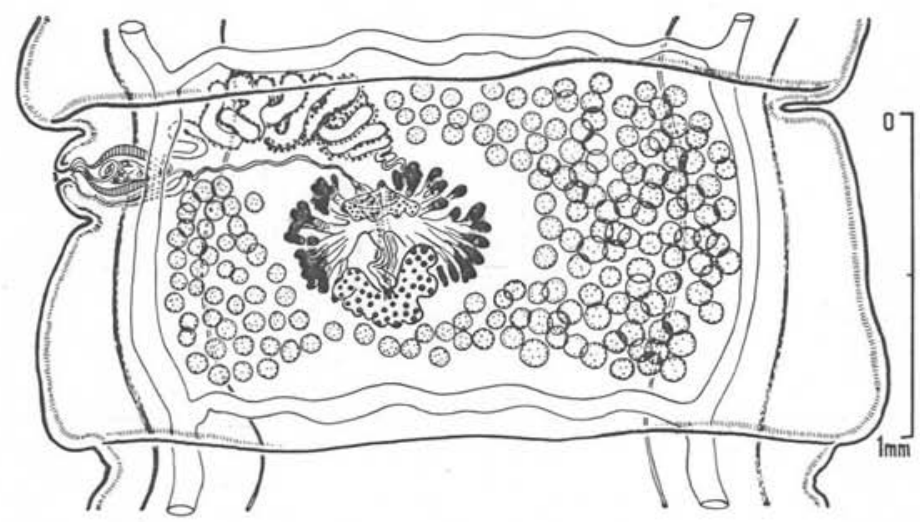

Fig. 3. - Anneau mûr, vue ventrale

se différencient lentement en position sub-médiane; apparaissent d'abord les ébauches vitello-ovariennes simultanément avec les canaux génitaux mâles et femelles; les ébauches testiculaires ne deviennent visibles au moins qu'à partir du $100-150^{\circ}$ anneau.

Anneaux mûrs (cf. fig. 3).

Taille moyenne : $2.500 \mu \times 900-1.000 \mu$. Rapport $\frac{\text { longueur }}{\text { largeur }}=\frac{1}{2,5}$. Forme trapézoïdale à bords latéraux convexes.

\section{Système génital mâle.}

Testicules: Nombreux: 100 à 190, sphériques ou légèrement ovalaires. Taille : 65 à $80 \mu$ de diamètre. Disposés en deux couches, plutôt dorsalement ; le champ testiculaire limité par le canal osmo-régulateur ventral occupe dans l'anneau tout l'espace laissé libre par les organes femelles et les conduits sexuels. La portion antiporale du champ dépasse antérieurement la limite for- 
mée par le bord antérieur de l'ovaire, tandis que sa portion porale moins étendue et moins fournie (1/3 à $1 / 4$ du nombre total des testicules) ne dépasse pas les canaux génitaux; les deux portions sont réunies l'une à l'autre en arrière de l'ovaire par une rangée de quelques testicules disposés irrégulièrement. Les testicules persistent un certain temps dans les anneaux gravides en notre possession, entre les capsules ovifères.

Canal déférent: Très long, il décrit de très nombreuses sinuosités dans toute la moitié porale de l'anneau en avant de l'ovaire et du champ testiculaire. Très dilaté (26-50 $\mu$ de diamètre), il joue le rôle d'une vésicule séminale externe. Il est entouré de cellules glandulaires relativement volumineuses et plus colorables.

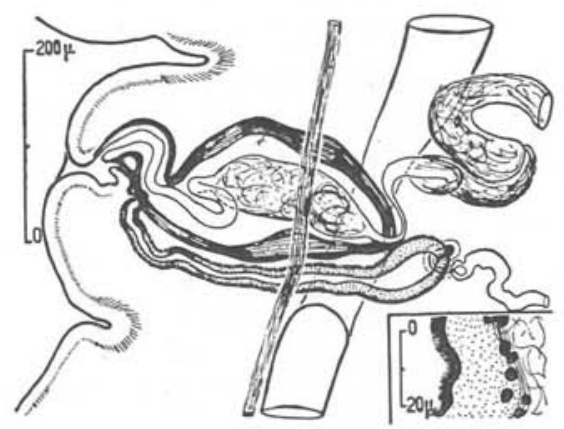

Fig. 4. - Atrium génital et terminaison des canaux sexuels, mâle et femelle. Dans le cartouche, détail de la paroi vaginale.

Poche du cirre (cf. fig. 4): Bien développée, elle se situe parallèlement au bord antérieur de l'anneau, atteignant toujours ou dépassant le canal osmorégulateur ventral. Taille : $310 \times 160 \mu$. Sa portion porale est fine et sinueuse, de parois musculeuses minces, et contient le cirre; sa portion antiporale est vésiculeuse, ovoïde, allongée, de parois musculeuses épaisses (25-30 $\mu$ ), et contient la vésicule séminale interne également ovoïde et non repliée sur ellemême. Le cirre est long et mince $(240 \times 13 \mu)$ et glabre ; toujours observé invaginé, il montre une forme de simple tube sinueux, dans la partie porale de la poche à parois musculeuses amincies.

La glande prostatique est réduite dans la partie antérieure de la poche du cirre.

L'atrium génital : Unilatéral, situé du côté droit ou gauche du strobile, au niveau d'une saillie convexe bien figurée de la paroi (longue de $300 \mu \times 150 \mu$ de large en moyenne), individualisée par deux sillons; sa cavité est petite, dépourvue d'ornementation cuticulaire ; l'ouverture vaginale s'ouvre en arrière de l'ouverture mâle. 


\section{Système génital femelle.}

Il occupe une position sub-centrale (le champ testiculaire antiporal est nettement plus important que le champ poral).

Ovaire : Formé de deux lobes plus ou moins confondus et très profondément digités, les digitations pouvant à leur tour se dichotomiser. Les deux lobes sont réunis par un isthme étroit dont l'axe transversal est légèrement oblique, l'ensemble de l'ovaire étant légèrement tourné du côté poral.

Taille de l'ovaire : $450 \mu$ de haut $\times 600 \mu$ de large.

Vitellogène: Postéro-dorsal à l'ovaire. Réniforme à concavité antérieure à l'état jeune.

Réceptacle séminal : Présent. Ovoïde, à parois minces, situé à peu près au niveau de l'isthme ovarien. Taille : $80 \times 100 \mu$.

Vagin : Issu du petit réceptacle séminal, son tube long de $500 \mu$ environ $\times$ 15-20 $\mu$ de diamètre décrit un arc de cercle régulier en se dirigeant vers la portion copulatrice terminale qu'il aborde par l'intermédiaire d'un étroit pertuis.

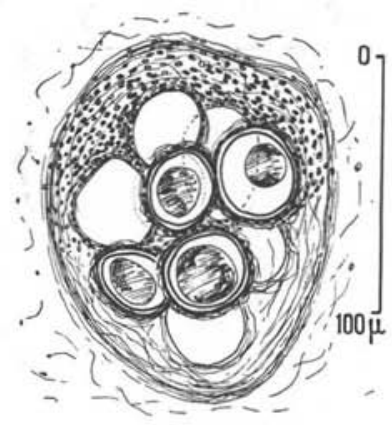

Fig. 5. - Capsule ovifère

La portion copulatrice du vagin plus longue que la poche du cirre franchit toujours légèrement le canal osmo-régulateur ventral. Taille : $420 \mu \times 18$ à $50 \mu$ de diamètre maximal à son extrémité antiporale où la paroi épaissie $(13-15 \mu)$ de nature musculeuse ménage une lumière bordée d'une cuticule épaisse de $2 \mu$, garnie d'une très fine ciliature dense, longue de $2-3 \mu$ (cf. fig. 4) qui n'est visible que sur coupes histologiques.

Utérus: L'utérus jeune se dichotomise rapidement dans tout le parenchyme ventralement par rapport aux testicules. Il ne persiste que dans une vingtaine d'anneaux et disparaît pour être remplacé par des capsules ovifères parenchymateuses pluriovulées.

Capsules ovifères (cf. fig. 5) : Très nombreuses (160 par anneau), elles mesurent 80 à $110 \mu-130 \mu$ de diamètre. Sphériques, mais plus ou moins déformées par pression réciproque, elles contiennent de 6 à 12 œufs de 25,5-38 $\times 25,5$ $29 \mu$ de diamètre. 
Il est vraisemblable que ces dimensions puissent s'accroître dans les derniers anneaux très mûrs de la chaîne (qui nous manquent). Les œufs sont insuffisamment mûrs pour que nous ayons été capables de décrire leur organisation interne.

\section{Discussion}

Ce Cestode possède les caractères essentiels suivants :

1) scolex inerme ;

2) pores génitaux unilatéraux antérieurs ;

3) conduits sexuels passant indifféremment à la face dorsale ou ventrale du nerf, et généralement entre les canaux excréteurs longitudinaux, le canal ventral pouvant les franchir dorsalement avec une fréquence variable suivant les strobiles ;

4) système excréteur de quatre vaisseaux longitudinaux, anastomosés deux à deux par des canaux transverses; un réseau dorsal réduit, de fins canalicules anastomosés ;

5) testicules très nombreux, ne formant qu'un champ unique de part et d'autre et en arrière de l'ovaire ;

6) ovaire sub-médian (très légèrement poral) ;

7) utérus en sac transverse fugace, se résolvant en capsules ovifères parenchymateuses pluriovulées, ne dépassant pas latéralement les canaux excréteurs ventraux.

Ce Plathelminthe correspond presqu'exactement, dans la sous-famille des Linstowinae Fuhr., 1907 (Anoplocephalidae Fuhr., 1907), si nous excluons Multicapsiferina Furm., 1921 à capsules uniovulées et Linstowia Zschokke, 1899 à pores génitaux alternes, aussi bien à la définition du genre Inermicapsifer Janicki, 1910 qu'à celle du genre Thysano:aenia Beddard, 1911.

Néanmoins, il y a lieu de faire remarquer que :

$1^{\circ}$ les Inermicapsifer s'en différencieraient :

a) par la position très porale d'un ovaire dans un anneau généralement beaucoup plus large que long (bien qu'I. madagascariensis, par exemple, puisse constituer une exception à cette règle) ;

b) par l'envahissement du proglottis dans sa totalité par les capsules ovifères qui franchissent latéralement le canal excréteur ventral ;

c) par la nature des hôtes (Rongeurs et Hyracoïdes) ;

d) par la répartition géographique (Afrique continentale) ;

$2^{\circ}$ l'unique représentant actuel du genre Thysanotaenia s'en différencierait uniquement : 
a) par la disposition dorsale des conduits sexuels par rapport aux canaux excréteurs ;

b) l'ornementation épineuse de l'organe copulateur mâle ;

c) la non-extension du champ testiculaire antiporal en avant de la limite constituée par le bord antérieur de l'ovaire.

Logiquement en conséquence, si l'on s'en tient scrupuleusement aux définitions actuelles, notre Cestode est inclassable. Dès lors, il deviendrait nécessaire de créer pour lui un genre nouveau en remarquant toutefois que ce genre neuf créerait un trait d'union très étroit entre les deux genres précités et ferait envisager ipso facto la synonymie des trois genres au profit du plus ancien (Inermicapsifer).

Mais l'identité des hôtes (Lémuriens) et de la localisation géographique (Madagascar) où ont été trouvés $T$. lemuris et notre Cestode nous porte à reconsidérer plus attentivement les caractères mêmes de $T$. lemuris et à envisager l'identité des deux espèces, solution la plus satisfaisante à notre avis. Toute l'anatomie est strictement identique, compte tenu des lacunes qui persistent dans la connaissance de cette espèce, pourtant déjà décrite deux fois. Cette similitude nous empêche par conséquent d'envisager l'existence d'un second Thysanotaenia différent de $T$. lemuris.

La disparité essentielle réside dans la position réciproque des canaux excréteurs et des conduits génitaux, position précisée par Joyeux et Baer (1927), qu'ils énoncent comme fixe.

Envisager la synonymie nous conduit donc à retenir deux éventualités :

$1^{\circ}$ Joyeux et Baer ont bien décrit les Cestodes qu'ils ont étudiés tandis que nous avons observé des exemplaires aberrants de la même espèce.

$2^{\circ}$ Ces auteurs, lors de leur redescription, n'ont pas eu la possibilité d'observer la réalité de certains détails.

a) Considérons la première proposition.

Nous avons observé personnellement plusieurs strobiles à divers stades de maturité résultant vraisemblablement d'infestations multiples. On doit donc admettre que Thysanotaenia présente indifféremment les deux possibilités de disposition, avec prédominance de la position intercalaire des canaux génitaux par rapport aux canaux excréteurs.

b) Reste la seconde proposition.

Elle n'est pas impossible a priori, car elle peut s'expliquer de deux façons :

a) Les auteurs ne précisent pas dans leur texte s'ils ont contrôlé avec attention ce détail anatomique. Peut-être ont-ils transcrit simplement le texte original de Beddard : « both of these (genital) tubes lie on the same side of the « lateral excretory vessels, if we are to regard the ovary as ventral, they lie « to the dorsal side ». Beddard n'ayant entrevu que le vaisseau ventral sur 
ses coupes histologiques, ce texte signifie que les canaux génitaux sont dorsaux par rapport aux canaux excréteurs ventraux et à l'ovaire; leur position reste inconnue vis-à-vis du canal excréteur dorsal.

$\beta)$ Beaucoup plus vraisemblablement, n'ayant examiné qu'un petit nombre d'anneaux, sont-ils tombés sur une séquence offrant cette seule disposition.

Restent les détails morphologiques plus mineurs.

Beddard, qui a observé des cirres évaginés, facteur particulièrement favorable d'une bonne description, n'y mentionne pas d'épines; nous sommes ici enclins à penser à une erreur d'interprétation de Joyeux et Baer qui signalent leur présence (1) à moins qu'ici encore puissent exister les deux possibilités, ce qui paraît peu vraisemblable ; notons, par contre, que la ciliature vaginale est bien visible et que ce détail, plus difficile à percevoir en principe qu'une spinulation de cirre même fine, nous met en accord avec la redescription de 1927.

Enfin, le champ testiculaire antiporal, s'il ne dépasse pas antérieurement la limite du bord de l'ovaire pour Joyeux et Baer, est décrit comme tel (p. 1003) par Beddard. Le nombre de testicules élevé jusqu'à 180 n'est pas à lui seul un facteur discriminant suffisant.

Devant ces imprécisions et ces doutes, nous préférons, au moins provisoirement, considérer comme identiques nos exemplaires, ceux de Joyeux et Baer, et l'espèce de Beddard. En conséquence, le genre Thysanotaenia ne se distingue plus d'Inermicapsifer que par le non franchissement des canaux excréteurs par les capsules parenchymateuses dans les anneaux gravides.

L'espèce 1. madagascariensis (Davaine, 1870), aux multiples synonymies précisées par Baer en 1956, ainsi que I. setii Janicki, 1910, par la position sub-médiane de leurs ovaires, fournissent le chaînon reliant le genre Thysanotaenia au genre Inermicapsifer; la question demeure posée de savoir si le seul caractère constitué par le non franchissement des canaux excréteurs par les capsules parenchymateuses constitue un caractère de discrimination générique de valeur suffisante car il est possible que, sur des fragments de strobiles assez longs de divers Inermicapsifer, la recherche systématique de la position respective des canaux excréteurs et des canaux sexuels révèle de temps à autre une variation de la régularité classique. La synonymie des deux genres Inermicapsifer et Thysanotaenia ne pourra être déduite que de recherches complémentaires effectuées en ce sens.

(1) Nos exemplaires personnels, fixés en bon état, nous ont donné des préparations démonstratives. 


\section{BIBLIOGRAPHIE}

Bedpard (F. E.), 1911. - Contribution to the anatomy and systematic arrangement of the Cestoidea. II. On two Cestodes from mammals. Proceedings of the General Meetings for Scientific Business of the Zoological Society of London, p. 994-1018.

- 1912. - On a species of Inermicapsifer from the hyrax and on the genera Zschokkella, Thysanotaenia and Hyracotaenia. Proceedings of the General Meetings for scientific Business of the Zoological Society of London, p. 576-607.

Baer (J.-G.), 1925. - Some Cestoda deseribed by Beddard, 1911-1920. Ann. Trop. Med. Parasit., 19, p. 1-22.

- 1927. - Monographie des Cestodes de la famille des Anoplocephalidae. Balletin Biologique de France et de Belgique, supplément X, 61, p. 1-241.

1935. - Etude de quelques Helminthes de Lémuriens. Revue Suisse de Zoologie, 42, p. 275-291.

1956. - The taxonomic position Taenia madagascariensis Davaine, 1870, a tapeworm parasite of Man and Rodents. Annals of Tropical Medicine and Parasitology, 1956, 50, p. 152-156.

1925. - Some Cestoda described by Beddard, 1911-1920. Ann. Trop. Med. Parasit., 19, p. 1-22.

Joyeux (C.) et BAER (J.-G.), 1927. - Etude de quelques Cestodes provenant des colonies françaises d'Afrique et de Madagascar. Ann. Parasit. hum. et comp., 5, p. 27-36.

MaноN (J.), 1954. - Contribution to the Helminth fauna of tropical Africa. Tapeworms from the belgian Congo. Ann. Musée Royal du Congo Beige, C. Zoologie, série V, 1 (2), p. 137-264.

SpassKy (A.-A.), 1951. - Anoplocephalata, Helminthes rubannés des animaux domestiques et sauvages. Osnovy tsestodologii, 1, p. 596-624.

(Travail du Laboratoire de Parasitologie de la Faculté de Médecine et de Pharmacie de Lille, Directeur : Professeur J. Biguet). 